\title{
Prevalência de asfixia perinatal em recém-nascidos de termo em maternidade de referência terciária e principais disfunções orgânicas associadas
}

\section{Prevalence of perinatal asphyxia in new born of term in maternity of tertiary reference and major associated organic dysfunctions}

Ionara Lucena Machadoํ. Maria Francielze Holanda Lavor ${ }^{2}$.

1 Médica pediatra, residente do Programa de Neonatologia, Maternidade Escola Assis Chateubriand (MEAC), Fortaleza, Ceará, Brasil. 2 Médica pediatra e neonatologista, Mestra em Saúde Pública pela Universidade Federal do Ceará (UFC). Médica da Maternidade Escola Assis Chateubriand (MEAC), Fortaleza, Ceará, Brasil.

\section{RESUMO}

Objetivo: conhecer a prevalência de asfixia perinatal e encefalopatia hipóxico isquêmica em recém-nascidos de termo no Serviço de Neonatologia de uma maternidade pública terciária do município de Fortaleza. Metodologia: foi realizado um estudo transversal, retrospectivo de dados secundários coletados nos prontuários dos recém-nascidos de termo com Apgar menor que sete no quinto minuto de vida. Período: 1 de março a 31 de agosto de 2016. Resultados: ocorreram 2.586 nascimentos, 1.919 foram de recémnascidos termo e destes, dezenove apresentaram asfixia. Oito pacientes foram excluídos por malformação congênita. A prevalência de asfixia observada foi de 5,7 por 1000 nascimentos a termo. Da amostra incluída, a frequência de encefalopatia foi de 54\%. A taxa de óbito foi de $45 \%$. Encontramos alteração de enzimas cardíacas em $82 \%$ dos casos e em $36 \%$ regurgitação tricúspide. Apresentaram função renal alterada $27 \%$ e oligúria 45\%. Necessitaram de ventilação mecânica 64\%. Enzimas hepáticas estavam alteradas em 54\% dos casos. Os pacientes com encefalopatia grave apresentaram disfunção de dois ou mais sistemas e todos foram a óbito. Conclusão: a prevalência de asfixia encontrada no estudo está dentro do esperado para uma maternidade de referência.

Palavras-chave: Recém-nascido. Asfixia neonatal. Hipóxia encefálica.

\section{ABSTRACT}

Objective: To know the prevalence of perinatal asphyxia and ischemic hypoxic encephalopathy in term newborns in the Neonatal Service of a tertiary public maternity hospital in the city of Fortaleza. Methods: A cross-sectional, retrospective study of secondary data collected in the records of term newborns with Apgar less than seven in the fifth minute of life was performed. Results: There were 2,586 births, 1,919 were of term newborns and of these nineteen presented asphyxia. Eight patients were excluded due to congenital malformation. The prevalence of asphyxia observed was 5.7 per 1000 full-term births. From the included sample, the frequency of encephalopathy was $54 \%$. The death rate was $45 \%$. We found cardiac enzyme alterations in $82 \%$ of the cases and in $36 \%$ tricuspid regurgitation. They had altered renal function $27 \%$ and oliguria $45 \%$. They needed mechanical ventilation $64 \%$. Hepatic enzymes were altered in $54 \%$ of cases. Patients with severe encephalopathy had dysfunction of two or more systems and all died. Conclusion: The low prevalence of asphyxia can be explained by the fact that it is a reference maternity.

Keywords: Infant newborn. Asphyxia neonatorum. Hypoxia brain.

Autor correspondente: Ionara Lucena Machado, Rua Leonardo Mota, 1515, apartamento 1002, Aldeota, Fortaleza, Ceará. CEP: $60170-041$. Telefone: +55 85 99853-3902. E-mail: ionaralucena@me.com

Conflito de interesses: Não há qualquer conflito de interesses por parte de qualquer um dos autores.

Recebido em: 15 Dez 2016; Revisado em: 20 Fev 2017; Aceito em: 05 Abr 2017. 


\section{INTRODUÇÃO}

À nível global estima-se que as principais causas de mortalidade neonatal sejam prematuridade, infecção e asfixia. ${ }^{1}$ No Brasil, centros isolados enfatizam a asfixia perinatal como uma das principais causas da mortalidade neonatal precoce, com variação em sua incidência conforme os critérios utilizados. ${ }^{2}$

A asfixia perinatal é uma condição de comprometimento da troca gasosa sanguínea durante o período intraparto que, se persistir, leva a hipoxemia e hipercapnia progressivas com acidose metabólica. ${ }^{3}$ A encefalopatia hipóxico-isquêmica (EHI) constitui a conseqüência mais grave da asfixia perinatal e ocorre em mais ou menos $35 \%$ dos RN que a apresentam. ${ }^{4}$

Anualmente, há de dois a quatro recém-nascidos $(\mathrm{RN})$ com EHI para cada 1.000 nascimentos vivos a termo e a taxa de mortalidade dos RN asfixiados que desenvolvem EHI é de $15 \%$ a $25 \% .^{4}$

Os estágios de comprometimento neurológico para classificação de EHI foram descritos por Sarnat e Sarnat em 1976 e são utilizados na pratica clínica até o momento. O paciente é classificado em três estágios conforme o nível de consciência, o tônus muscular, a postura, os reflexos tendinosos, a presença ou não de mioclonias e a alteração das funções autonômicas. ${ }^{5}$

As convulsões podem estar presentes como única manifestação neurológica após insulto asfíxico, geralmente se iniciam nas primeiras 24 horas de vida do recém-nascido, são prolongadas e resistentes ao tratamento anticonvulsivante. ${ }^{6}$

Os critérios para o diagnóstico de asfixia variam nos artigos encontrados na literatura.

Em 1953, Virgínia Apgar propôs um índice para avaliar a condição física dos recém-nascidos logo após o nascimento. ${ }^{7}$ O Apgar é determinado em um e cinco minutos após o parto e é, portanto, uma maneira rápida de avaliar a condição física dos recém-nascidos. A pontuação de cinco minutos tem sido considerada como o melhor preditor de sobrevida na infância. ${ }^{8}$

De acordo com a Classificação Internacional de Doenças da Organização Mundial de Saúde (CID 10), asfixia perinatal grave é definida com Apgar de primeiro minuto de 0-3. Asfixia durante o parto leve e moderada é classificada com Apgar de primeiro minuto de vida de $4-7 .{ }^{9}$

O estudo sobre o tema é extremamente importante tendo em vista ser uma das principais causas de mortalidade e morbidade neonatais. O número elevado de mortes por asfixia intraparto, sobretudo de crianças com peso adequado ao nascer e em gravidez de baixo risco, demonstra o grande potencial de evitabilidade dessas mortes, o que justifica a relevância deste trabalho e demonstra a importância de uma assistência adequada com equipe treinada e atualizada em reanimação neonatal.

Este artigo visa identificar a prevalência de asfixia perinatal e encefalopatia hipóxico isquêmica em recém-nascidos de termo no Serviço de Neonatologia de uma maternidade pública terciária do município de Fortaleza, assim como relatar a presença de disfunção orgânica nos recém-nascidos de termo com asfixia.

\section{MATERIAL E MÉTODOS}

Foi realizado um estudo transversal, retrospectivo de dados secundários, onde os dados foram coletados nos prontuários de recém-nascidos termo na Maternidade Escola Assis Chateaubriand, em Fortaleza - Ceará; maternidade pública, classificada pelo gestor do Sistema Único de Saúde como terciária, pertencente à Universidade Federal do Ceará (UFC), no período de 1 de março a 31 de agosto de 2016, através de questionário simples.

O protocolo de pesquisa foi aprovado pelo Comitê de Ética em Pesquisa da instituição, estando de acordo com os critérios estabelecidos pela Declaração de Helsinki com as suas modificações. Não houve necessidade de consentimento informado por parte dos familiares, pois se tratou de levantamento de dados secundários coletados através de prontuários.

Para análise da prevalência de asfixia na população estudada, foram incluídos apenas os recém-nascidos de termo (RNT) que apresentaram Apgar de 5 minutos de vida $<7$, baseado na classificação da organização mundial de saúde usando o minuto 5 para maior acurácia dos casos, conforme observado na literatura revisada; ou a presença de convulsões nas primeiras 24 horas de vida, excluídas outras causas, e a partir destes, analisado a prevalência de encefalopatia hipóxico isquêmica de acordo com a classificação de Sarnat e Sarnat e a prevalência do acometimento orgânico sistêmico nos recém nascidos com asfixia. Sendo critério de exclusão a presença de malformação congênita.

As variáveis do processo de atenção relacionadas com a morbidade foram as funções dos diversos órgãos que podem estar comprometidos durante a asfixia. Foram avaliadas através de exames realizados nas primeiras 72 horas de vida.

A tabulação e análise dos dados de forma isolada e a confecção dos gráficos das variáveis foi realizada através do programa Microsoft Office Excel 2007.

O programa de pstatística utilizado foi o programa EpiInfo 7 (CDC, Atlanta, USA) sendo realizado o Teste Exato de Fisher para testar as variáveis analisadas. Para todos os testes analisados, adotou-se o valor de significância estatística menor ou igual a $5 \%(\mathrm{p} \leq 0,05)$.

\section{RESULTADOS}

No período de 01 de março a 31 de agosto de 2016 ocorreram 2.586 nascimentos na Maternidade Escola Assis Chateubriand. Desse total de nascimentos, 1.919 foram de recém-nascidos 
de termo, e destes, 19 apresentaram asfixia. Foi necessário excluir 8 pacientes por apresentarem malformação congênita: mielomeningocele (1), microcefalia por zyka (1), anencefalia (2), encefalocele occipital (1), síndrome de Patau (1), cardiopatia complexa (1) e com malformações múltiplas (1).

A prevalência de asfixia observada neste estudo foi de 5,7 por 1.000 nascimentos a termo. Dos 1.919 nascimentos a termo evoluíram com encefalopatia hipóxico-isquemica 6 RN, ou seja, a prevalência de encefalopatia observada neste estudo foi de 3,1 por 1.000 nascimentos a termo.

Um recém-nascido foi incluído no estudo por apresentar convulsões precoces, sendo descartadas outras causas, apesar de Apgar de quinto minuto de 8. Este RN evoluiu com encefalopatia grave. Apenas 27\% tiveram Apgar de quinto minuto $0-3$. Todos os RN foram reanimados na sala de parto.

Dos pacientes asfixiados $91 \%$ foram admitidos em unidade de terapia intensiva. Por sua vez, $64 \%$ necessitaram de ventilação mecânica. A síndrome de aspiração meconial esteve presente em $36 \%$ dos casos. Constatou-se que $27 \%$ apresentaram hipertensão pulmonar.

Em relação ao acometimento cardíaco, o valor encontrado da CKMB (enzima creatinoquinase fração MB) variou de 34 a 3579 U/l. A troponina teve variação entre 6,6 a 924.

O acometimento hepático foi analisado através das enzimas transaminase glutâmico oxalacética que variou de 36 a 109 U/1 e transaminase glutamicopirúvica com variação de 21 a $226 \mathrm{U} / 1$.

Em relação a função renal foi dosado o valor da creatinina que variou de $0,4 \mathrm{a} 1,3 \mathrm{mg} / \mathrm{dl}$. A diurese variou de $0,2 \mathrm{a} 1,8 \mathrm{ml} /$ $\mathrm{kg} / \mathrm{h}$ nas primeiras $72 \mathrm{~h}$.

Dentre os recém-nascidos que evoluíram a óbito, a maioria teve diagnóstico de encefalopatia ( $80 \%$ vs $20 \%$; $\mathrm{p}=0,175)$. Esse dado não foi significativamente estatístico (Tabela 1).

Em relação à evolução para óbito naqueles pacientes com diagnóstico de encefalopatia, todos apresentavam encefalopatia grave com $p=0,06$. Esse dado não foi significativamente estatístico (Tabela 2).

Em relação à presença de oligúria (diurese $\leq 0,5 \mathrm{ml} / \mathrm{kg} / \mathrm{h}$ ), a maioria dos recém-nascidos teve diagnóstico de encefalopatia ( $83 \%$ vs $17 \%$; $p=0,06)$. Esse dado não foi significativamente estatístico (Tabela 3 ).

Dentre os recém-nascidos que apresentaram necessidade de ventilação mecânica, a maioria teve diagnóstico de encefalopatia $(71 \%$ vs $29 \%$; $=0,196)$. Esse dado não foi significativamente estatístico (Tabela 4).

Dentre os recém-nascidos que apresentaram hipertensão pulmonar, todos tiveram diagnóstico de encefalopatia grave com $p=0,4$. Esse dado não foi significativamente estatístico (Tabela 5).
Tabela 1. Comparação entre óbito e encefalopatia.

\begin{tabular}{lllll}
\hline \multirow{2}{*}{ ÓBITO } & \multicolumn{2}{l}{ PRESENÇA DE } & & \\
\cline { 2 - 3 } & ENCEFALOPATIA & TOTAL & Valor $\boldsymbol{p}^{*}$ \\
\cline { 2 - 3 } NÃO & \multicolumn{2}{c}{ SIM } & & \\
SIM & $4(67 \%)$ & $2(33 \%)$ & 6 & 0,175 \\
TOTAL & $1(20 \%)$ & $4(80 \%)$ & 5 & \\
\hline
\end{tabular}

*Valor p para as diferenças de médias entre os grupos pelo Teste Exato de Fisher.

Tabela 2. Comparação entre taxa de óbito e encefalopatia grave.

\begin{tabular}{|c|c|c|c|c|}
\hline \multirow[t]{2}{*}{ ÓBITO } & \multicolumn{2}{|c|}{$\begin{array}{l}\text { PRESENÇA DE } \\
\text { ENCEFALOPATIA } \\
\text { GRAVE (GRAU 3) }\end{array}$} & \multirow[t]{2}{*}{ TOTAL } & \multirow[t]{2}{*}{ Valor $p^{*}$} \\
\hline & NÃO & SIM & & \\
\hline NÃO & $2(100 \%)$ & 0 & 2 & \multirow{3}{*}{0,06} \\
\hline SIM & 0 & $4(100 \%)$ & 4 & \\
\hline TOTAL & 2 & 4 & 6 & \\
\hline
\end{tabular}

*Valor p para as diferenças de médias entre os grupos pelo Teste Exato de Fisher.

Tabela 3. Comparação entre diurese e encefalopatia.

\begin{tabular}{|c|c|c|c|c|}
\hline \multirow[t]{2}{*}{ DIURESE } & \multicolumn{2}{|c|}{$\begin{array}{l}\text { PRESENÇA DE } \\
\text { ENCEFALOPATIA }\end{array}$} & \multirow[t]{2}{*}{ TOTAL } & \multirow[t]{2}{*}{ Valor $p^{*}$} \\
\hline & NÃO & SIM & & \\
\hline$>0,5 \mathrm{ml} / \mathrm{kg} / \mathrm{h}$ & $4(80 \%)$ & $1(20 \%)$ & 5 & \multirow{2}{*}{0,06} \\
\hline$\leq 0,5 \mathrm{ml} / \mathrm{kg} / \mathrm{h}$ & $1(17 \%)$ & $5(83 \%)$ & 6 & \\
\hline TOTAL & 5 & 6 & 11 & \\
\hline
\end{tabular}

*Valor p para as diferenças de médias entre os grupos pelo Teste Exato de Fisher.

Tabela 4. Comparação entre necessidade de ventilação mecânica e encefalopatia.

\begin{tabular}{lllll}
\hline \multirow{2}{*}{$\begin{array}{l}\text { NECESSIDADE } \\
\text { DE VENTILAÇÃO }\end{array}$ MECÂNICA } & \multicolumn{2}{l}{ PRESENÇA DE } & & \\
\cline { 2 - 3 } & ENCEFALOPATIA & \multirow{2}{*}{ TOTAL } & Valor $\boldsymbol{p}^{*}$ \\
\hline NÃO & $3(75 \%)$ & $1(25 \%)$ & 4 & \\
SIM & $2(29 \%)$ & $5(71 \%)$ & 7 & 0,196 \\
TOTAL & 5 & 6 & 11 & \\
\hline
\end{tabular}

*Valor p para as diferenças de médias entre os grupos pelo Teste Exato de Fisher. 
Tabela 5. Comparação entre presença de hipertensão pulmonar e encefalopatia grave.

\begin{tabular}{|c|c|c|c|c|}
\hline \multirow[t]{2}{*}{$\begin{array}{l}\text { HIPERTENSÃO } \\
\text { PULMONAR }\end{array}$} & \multicolumn{2}{|c|}{$\begin{array}{l}\text { PRESENÇA DE } \\
\text { ENCEFALOPATIA } \\
\text { GRAVE (GRAU 3) }\end{array}$} & \multirow[t]{2}{*}{ TOTAL } & \multirow[t]{2}{*}{ Valor $p^{*}$} \\
\hline & NÃO & SIM & & \\
\hline NÃO & $2(50 \%)$ & $2(50 \%)$ & 4 & \multirow{3}{*}{0,4} \\
\hline SIM & 0 & $2(100 \%)$ & 2 & \\
\hline TOTAL & 2 & 4 & 6 & \\
\hline
\end{tabular}

*Valor p para as diferenças de médias entre os grupos pelo Teste Exato de Fisher.

\section{DISCUSSÃO}

A prevalência da asfixia perinatal em RNT foi de 5,7 por 1.000 nascimentos, ou seja, $0,57 \%$. Cloherty et $\mathrm{al}^{10}$ relata ocorrência de asfixia em $0,5 \%$ de RN com mais de 36 semanas. Hall et $\mathrm{al}^{11}$ mostrou resultado similar de 4,6 por 1.000 nascimentos em estudo retrospectivo acompanhando 15.964 nascimentos. Cruz et al ${ }^{12}$ analisando RNT encontrou uma prevalência de 3,2 por 1000 nascimentos a termo.

$\mathrm{Na}$ literatura revisada a prevalência de asfixia perinatal variou de 3 a 28 por 1000 nascidos vivos. Essa variação ampla se deve a falta de um único consenso nos indicadores de asfixia como também as diferenças regionais na assistência. Na África, Ugwu et al ${ }^{13}$ mostrou uma prevalência de asfixia de 28 por 1000 nascimentos.

A encefalopatia hipóxico isquêmica constitui a consequência mais grave da asfixia. Quando analisados os $11 \mathrm{RN}$ que apresentaram asfixia perinatal a frequência de encefalopatia foi de $54 \%$. Cruz et al ${ }^{12}$ relatou resultado similar de 53\%. Dos pacientes com asfixia 36\% apresentaram encefalopatia grave. Simovic et $\mathrm{al}^{14}$ encontrou $21 \%$ de casos com encefalopatia grave.

A taxa de óbito foi de $45 \%$ em relação aos pacientes com asfixia e destes óbitos $60 \%$ foram precoces, ou seja, com menos de 7 dias de vida. Vários estudos relatam o grande impacto da asfixia perinatal na mortalidade neonatal. Simovic et $\mathrm{al}^{14}$ relatou taxa de $23,6 \%$ de óbito em RNT asfixiados com $53,8 \%$ dos casos ocorrendo precocemente. Sadoh et $\mathrm{al}^{15}$ observou taxa de óbito de $20 \%$ em asfixiados.

\section{REFERÊNCIAS}

1. Lawn JE, Cousens S, Zupan J. 4 million neonatal deaths: When? Where? Why? Lancet. 2005;365(9462):891-900.

2. Daripa M, Caldas HM, Flores LP, Waldvogel BC, Guinsburg R, Almeida MF. Asfixia perinatal associada à mortalidade neonatal precoce: estudo populacional dos óbitos evitáveis. Rev Paul Pediatr. 2013;31(1):37-45.

3. Gomella TL, Cunningham MD, Eval FG. Neonatologia, tratamento,
Encontramos alteração de enzimas cardíacas em $82 \%$ dos casos. Simovic et $\mathrm{al}^{14}$ observou disfunção cardíaca em $78 \%$ dos RNT com asfixia. Sadoh et $\mathrm{a}^{15}$ encontrou $32,5 \%$.

A regurgitação tricúspide significativa é outro marcador de isquemia miocárdica, sendo importante em prever mortalidade em neonatos com asfixia. Encontramos 36\% de regurgitação tricúspide nos pacientes asfixiados. Simovic et al ${ }^{14}$ encontrou regurgitação em $76,9 \%$ dos $\mathrm{RN}$ asfixiados que foram a óbito. Shah et $\mathrm{al}^{16}$ encontrou $62 \%$ de disfunção cardíaca.

A lesão renal aguda é uma importante complicação da asfixia. Dos pacientes analisados $27 \%$ apresentaram alteração de função renal. Sadoh et a ${ }^{15}$ relatou 35,3\% de lesão renal. Shah et $\mathrm{al}^{16}$ encontrou $70 \%$ de lesão renal em $\mathrm{RN}$ com asfixia grave.

O acometimento pulmonar pode ser demonstrado pela necessidade de ventilação mecânica. Em nosso estudo a prevalência foi de $63 \%$. Shah et $\mathrm{al}^{16}$ relatou necessidade de ventilação mecânica em $86 \%$ dos casos.

As enzimas hepáticas estiveram alteradas em 54\%. Chhavi et al $^{17}$ encontrou resultado similar de $56 \%$ de lesão hepática associada a asfixia. Shah et $\mathrm{al}^{16}$ observou $85 \%$ de acometimento hepático.

Observamos que os RN com encefalopatia grave apresentaram disfunção de 2 ou mais órgãos ou sistemas.

Dentre as relações examinadas, as que mais se aproximaram de relevância estatística foram: oligúria (diurese $\leq 0,5 \mathrm{ml} /$ $\mathrm{kg} / \mathrm{h}$ ) $\mathrm{x}$ encefalopatia com $\mathrm{p}=0,06$; necessidade de ventilação mecânica $\mathrm{x}$ encefalopatia com $\mathrm{p}=0,196$; evolução ao óbito $\mathrm{x}$ encefalopatia com $\mathrm{p}=0,175 \mathrm{e}$; evolução ao óbito $\mathrm{x}$ encefalopatia grave com $\mathrm{p}=0,06$.

\section{CONCLUSÃO}

A prevalência de asfixia encontrada no estudo está dentro do esperado para uma maternidade de referência. Os resultados não tiveram relevância estatística $(\mathrm{p} \geq 0,05)$, provavelmente devido ao número de pacientes da amostra. Torna-se necessário um estudo realizado em um período mais longo para se obter uma amostra significativa do serviço.

Recomendamos a manutenção de treinamento da equipe relacionada a assistência neonatal para prevenção da asfixia e a implantação de protocolo de Hipotermia terapêutica no serviço para tratamento da encefalopatia neonatal.

procedimentos, problemas no plantão, doenças e drogas. 6. ed. Rio de Janeiro: Revinter; 2012.

4. Brasil. Ministério da Saúde. Atenção à saúde do recém-nascido: guia para os profissionais de saúde. Volume 3. Brasília: Ministério da Saúde; 2011. Capítulo 28, Encefalopatia Hipóxico-Isquêmica; p.135-148.

5. Sarnat HB, Sarnat MS. Neonatal encephalopathy following fetal distress. Arch Neurol. 1976;33(10):696-705. 
6. Procianoy RS, Silveira RC. Síndrome hipóxico-isquêmica. J Pediatr. 2001;77(Supl 1):563-70.

7. Apgar V. A proposal for a new method of evaluation of the newborn infant. Curr Res Anesth Analg. 1953;32(4):260-7.

8. Casy BM, McIntire DD, Leveno KJ. The continuing value of the Apgar score for the assessment of newborn infants. N Engl J Med. 2001;344(7):467-71.

9. World Health Organization. ICD-10 version 2016. 2016 [Acesso em: 15 out 2016]. Disponível em: http://apps.who.int/classifications/ icd10/browse/2016/en

10. Cloherty JP, Eichenwald EC, Stark AR. Manual de neonatologia. 7. ed. Rio de Janeiro: Guanabara Koogan; 2015.

11. Hall DR, Smith M, Smith J. Maternal factores contributing to asphyxia neonatorum. J Trop Pediatr. 1996;42(4):192-5.

12. Cruz AC, Ceccon ME. Prevalência de asfixia perinatal e encefalopatia hipóxico-isquêmica em recém-nascidos de termo considerando dois critérios diagnósticos. Rev Bras Crescimento Desenvolvimento Hum. 2010;20(2):302-16.
13. Ugwu GI, Abedi HO, Ugwu EN. Incidence of birth asphyxia as seen in Central Hospital and GN Children's Clinic both in Warri Niger Delta of Nigeria: an eight year retrospective review. Glob J Health Sci. 2012;4(5):140-6.

14. Simovic AM, Prijic S, Knezevic J, Kosutic JL. Predictive value of biochemical, echocardiographic and eletrocardiographic markers in non surviving and surviving asphyxiated full-term newborns. Turk J Pediatr. 2014;56:243-9.

15. Sadoh WE, Eregie CO, NwanerI DU, Sadoh AE. The diagnostic value of both troponin $\mathrm{T}$ and creatinine kinase isoenzyme (CK-MB) in detecting combined renal and myocardial injuries in asphyxiated infants. Plos One. 2014;9(3):e91338.

16. Shah P, Riphagen S, Beyene J, Perlman M. Multiorgan dysfunction in infants with post asphyxial hypoxic-ischaemic encephalopathy. Arch Dis Child Fetal Neonatal. 2004;89(2):152-5.

17. Chhavi N, Zutshi K, Singh NK, Awasthi A, Goel A. Serum liver enzyme pattern in birth asphyxia associated liver injury. Pediatr Gastroenterol Hepatol Nutr. 2014;17(3):162-9.

\section{Como citar:}

Machado IL, Lavor MF. Prevalência de asfixia perinatal em recém-nascidos de termo em maternidade de referência terciária e principais disfunções orgânicas associadas. Rev Med UFC. 2018 jul-set;58(3):10-14. 